\title{
Bosonization theory of fermions interacting via a Coulomb potential in the limit $e^{2} \rightarrow \infty$
}

\author{
Tai-Kai Ng \\ Department of Physics, Hong Kong University of Science and Technology, Clear Water Bay Road, Kowloon, Hong Kong
}

(Received 11 June 1999; revised manuscript received 20 January 2000)

\begin{abstract}
We study in this paper the properties of a gas of fermions interacting via a scalar potential $v(q)$ $=4 \pi e^{2} / q^{2}$ for $q<\Lambda \ll k_{F}$ at dimensions larger than 1 , where $\Lambda \ll k_{F}$ is a high-momentum cutoff and $k_{F}$ is the Fermi wave vector. In particular, we shall consider the $e^{2} \rightarrow \infty$ limit, where charge fluctuations at wave vectors $q<\Lambda$ are forbidden. Within a bosonization approximation, effective actions describing the low-energy physics of the system are constructed, where we show that the system can be described as a Fermi liquid formed by chargeless quasiparticles, which has vanishing wave function overlap with the bare fermions in the system.
\end{abstract}

\section{INTRODUCTION}

In the last few years, there has been enormous interest in the study of $U(1)$ gauge theories of fermionic systems in dimensions higher than 1 , as a result of the appearance of effective gauge theories in the $t-J$ type models ${ }^{1}$ and also in the studies of the $\nu=\frac{1}{2}$ fractional quantum Hall state., ${ }^{2,3}$ In particular, the behavior of the systems in the regime where charge fluctuations are "confined" are of special interest. In this paper we shall apply a bosonization procedure to study a system of charge $e$ spinless fermions interacting with a scalar gauge field $\phi$ in dimensions higher than 1 , with effective long distance gauge field action $L_{\phi}=(\nabla \phi)^{2}$. The problem is equivalent to fermions interacting via a scalar potential $v(q)=4 \pi e^{2} / q^{2}$ for $q<\Lambda$, where $\Lambda \ll k_{F}$ is a highmomentum cutoff and $k_{F}$ is the Fermi wave vector. In particular, we are interested at the $e^{2} \rightarrow \infty$ limit where any nonuniform charge density fluctuations $\langle\rho(\vec{q})\rangle \neq 0$ with wave vector $q<\Lambda$ cost infinite electric field energy. As a result any physical state $|\psi\rangle$ that survives in this limit satisfies the constraint $\rho(\vec{q})|\psi\rangle=0$, where $\rho(\vec{q})$ is the charge density operator.

In this paper we shall show that within a bosonization approximation the projection to the physical states $|\psi\rangle$ where density fluctuations are forbidden can be carried out in the $e^{2} \rightarrow \infty$ limit, the bosonization approximation being a generalization of the usual bosonization method in one dimension to dimensions higher than $1 .^{4-7}$ Within this approximation an effective low-energy Lagrangian, which describes the particle-hole as well as the single-particle excitations in the system, can be constructed. In particular, the single-particle excitations are described by an operator that commutes with the charge operator $\rho(\vec{q})$, and has zero overlap with the bare fermion operators in the system. The ground state of the system can be described as a Fermi liquid of these singleparticle operators and corresponds to a kind of "marginal" Fermi liquid in the original fermion description. The organization of our paper is as follows. In Sec. II we shall outline our mathematical formulation of the bosonization procedure and the nature of the bosonization approximation will be explained. In Sec. III we shall study in detail the eigenstates and eigenvalues of the particle-hole excitation spectrum in the bosonization approximation for arbitrary values of coupling constant $e^{2}$. In particular, we shall show how the projection to the physical Hilbert space where charged excitations are forbidden is achieved in the $e^{2} \rightarrow \infty$ limit. In Sec. IV we shall consider the single-particle excitations, where "physical" single-particle operators that commute with density operators are constructed and the equations of motion for these single-particle operators are derived. An effective action in terms of the "physical" single-particle operators will be constructed and we shall show that the system can be described as a marginal Fermi liquid of the original fermions. Our results will be summarized in Sec. V where some further comments will be given.

\section{MATHEMATICAL FORMULATION}

We consider a gas of spinless fermions interacting via the scalar potential $v(q)=4 \pi e^{2} / q^{2}$ in momentum space. The Hamiltonian of the system is

$$
H=\sum_{\vec{k}} \epsilon_{\vec{k}} f_{\vec{k}}^{\dagger} f_{\vec{k}}+\frac{1}{2 L^{d}} \sum_{\vec{q} \neq 0, q<\Lambda} v(q) \rho(\vec{q}) \rho(-\vec{q}),
$$

where $\boldsymbol{\epsilon}_{\vec{k}}=(\vec{k})^{2} / 2 m$ and $f\left(f^{\dagger}\right) \vec{k}$ are fermion annihilation (creation) operators. We have set $\hbar=1$ to simplify notation. $\rho(\vec{q})=\sum_{\vec{k}} f_{\vec{k}+\vec{q} / 2}^{\dagger} f_{\vec{k}-\vec{q} / 2}$ is the density operator for the fermions. $L^{d}$ is the volume of the system. $\Lambda \ll k_{F}$ is a highmomentum cutoff. Notice that the Hartree $(\vec{q}=0)$ interaction energy does not appear in the Hamiltonian as in the usual Coulomb gas problem. The difference between our model and the usual Coulomb gas problem has to be emphasized here. In the usual Coulomb gas problem the high-momentum cutoff $\Lambda$ is taken to be infinity, or satisfies $\Lambda \gg k_{F}$. In this limit a Wigner crystal is expected to be formed [in three dimensions (3D)] when $e^{2}$ becomes large because the potential energy term becomes dominating at length scale $\sim k_{F}^{-1}$. In our problem, where $\Lambda \ll k_{F}$, the interaction is effective only at length scales much greater than the interparticle spacing and formation of a Wigner crystal is not warranted. To see this, we first neglect the kinetic energy term and assume that the fermions form a regular Wigner crystal state $\left|\psi_{W C}\right\rangle$. In this state $\rho(\vec{q})\left|\psi_{W C}\right\rangle \neq 0$ only when $\vec{q}$ is equal to a recip- 
rocal lattice vector (except for $\vec{q}=0$ ) and the potential energy of the state is zero as long as $\Lambda$ is less than the smallest reciprocal lattice vector $q_{1}$. For $\Lambda \ll k_{F}$ this is satisfied for any crystal structure with fermion density $\sim k_{F}^{d}\left(q_{1} \sim k_{F}\right)$ and the crystal ground state has a large degeneracy. As a result the exact ground state of the system depends strongly on the kinetic energy term and the system may remain in a liquid state. We note that the bosonization method is a natural tool to study the liquid state of the problem in this limit. ${ }^{6-8}$ The bosonization procedure can be formulated most easily by introducing the Wigner function operators $\rho_{\vec{k}}(\vec{q})$ $=f_{\vec{k}+\vec{q} / 2}^{\dagger} f_{\vec{k}-\vec{q} / 2}$. We shall work in the path-integral formulation where the Wigner operators are introduced in the action of the system at imaginary time through Langrange multiplier fields,

$$
\begin{aligned}
S= & \int_{0}^{\beta} d \tau\left[\sum_{\vec{k}} f_{\vec{k}}^{\dagger}(\tau)\left(\frac{\partial}{\partial \tau}+\epsilon_{\vec{k}}-\mu\right) f_{\vec{k}}(\tau)-\sum_{\vec{k}, \vec{q} \neq 0} i \lambda_{\vec{k}}(\vec{q}, \tau)\right. \\
& \times\left[\rho_{\vec{k}}(\vec{q}, \tau)-f_{\vec{k}+\vec{q} / 2}^{\dagger}(\tau) f_{\vec{k}-\vec{q} / 2}(\tau)\right] \\
& \left.+\frac{1}{2 L^{d}} \sum_{\vec{q} \neq 0, q<\Lambda, \vec{k}, \vec{k}^{\prime}} v(q) \rho_{\vec{k}}(\vec{q}, \tau) \rho_{\vec{k}^{\prime}}(-\vec{q}, \tau)\right]
\end{aligned}
$$

where $\mu$ is the chemical potential. $\lambda_{\vec{k}}(\vec{q})$ are Lagrange multiplier fields introduced to enforce the constraint that the

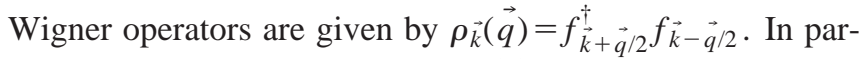
ticular, the original Hamiltonian (1) is recovered once the $\lambda_{\vec{k}}(\vec{q})$ field is integrated out.

Integrating out first the fermion fields $f\left(f^{\dagger}\right)$ we obtain an action in terms of the $\rho_{\vec{k}}(\vec{q})$ and $\lambda_{\vec{k}}(\vec{q})$ fields,

$$
\begin{aligned}
S= & \frac{1}{\beta} F_{0}-\operatorname{Tr} \ln \left[\hat{\imath}-\hat{G}_{o} \hat{\lambda}\right]-\sum_{\vec{k}, \vec{q} \neq 0, q<\Lambda} i \lambda_{\vec{k}}(\vec{q}) \rho_{\vec{k}}(\vec{q}) \\
& +\frac{1}{2 L^{d}} \sum_{\vec{q} \neq 0, q<\Lambda, \vec{k}, \vec{k}^{\prime}} v(q) \rho_{\vec{k}}(\vec{q}) \rho_{\vec{k}^{\prime}}(-\vec{q}),
\end{aligned}
$$

where $F_{0}$ is the free energy for a noninteracting Fermi gas. $\hat{G}_{0}$ and $\hat{\lambda}$ are infinite matrices in wave vector and frequency space, with matrix elements given by

$$
\left[\hat{G}_{0}\right]_{k, k^{\prime}}=\delta_{k, k^{\prime}} g_{0}(k), \quad g_{0}(k)=\frac{1}{i \omega_{n}-\xi_{\vec{k}}},
$$

and

$$
\begin{aligned}
{[\hat{\lambda}]_{k, k^{\prime}} } & =\frac{i}{\sqrt{\beta}} \lambda_{\left(\vec{k}+\vec{k}^{\prime}\right) / 2}\left(\vec{k}-\vec{k}^{\prime}, i \omega_{n}-i \omega_{n^{\prime}}\right) \\
& =\frac{i}{\sqrt{\beta}} \lambda_{\left(k+k^{\prime}\right) / 2}\left(k-k^{\prime}\right),
\end{aligned}
$$

where $k=\left(\vec{k}, i \omega_{n}\right)$ and $\xi_{\vec{k}}=\epsilon_{\vec{k}}-\mu$. The $\operatorname{Tr} \ln \left[1-G_{0} \lambda\right]$ term can be expanded in a power series of the $i \lambda_{\vec{k}}(\vec{q})$ field,

$$
\begin{aligned}
\operatorname{Tr} \ln \left[\hat{1}-\hat{G}_{0} \hat{\lambda}\right]= & -\operatorname{Tr}\left[\hat{G}_{0} \hat{\lambda}\right]-\frac{1}{2} \operatorname{Tr}\left[\hat{G}_{0} \hat{\lambda}\right]^{2}-\frac{1}{3} \operatorname{Tr}\left[\hat{G}_{0} \hat{\lambda}\right]^{3} \\
& +O\left(\hat{\lambda}^{4}\right) .
\end{aligned}
$$

Keeping terms to second order in $\hat{\lambda}$ (Gaussian approximation), we obtain

$$
\begin{aligned}
\operatorname{Tr} \ln \left[\hat{1}-\hat{G}_{0} \hat{\lambda}\right] & \sim \frac{1}{2 \beta} \sum_{k, q} g_{0}\left(k+\frac{q}{2}\right) g_{0}\left(k-\frac{q}{2}\right) \\
& \times \lambda_{k}(q) \lambda_{k}(-q) .
\end{aligned}
$$

Notice that the first order term in $\hat{\lambda}$ gives the usual Hartree self-energy and is excluded in the problem. The $i \lambda_{\vec{k}}(\vec{q})$ fields in action (3) can be integrated out in the Gaussian approximation, resulting in a quadratic action in terms of $\rho_{\vec{k}}(\vec{q})$ fields only. We obtain

$$
\begin{aligned}
S_{\rho}= & \frac{1}{2 L^{d}} \sum_{\vec{k}, \overrightarrow{k^{\prime}}, \vec{q}, i \omega_{n}}\left(-\frac{1}{\chi_{0 \vec{k}}\left(\vec{q}, i \omega_{n}\right)}\left(L^{d} \delta_{\vec{k}, \vec{k}^{\prime}}\right)+v(q)\right) \\
& \times \rho_{\vec{k}}\left(\vec{q}, i \omega_{n}\right) \rho_{\vec{k}^{\prime}}\left(-\vec{q},-i \omega_{n}\right),
\end{aligned}
$$

where

$$
\begin{aligned}
\chi_{0 \vec{k}}\left(\vec{q}, i \omega_{n}\right) & =\frac{1}{\beta} \sum_{i \Omega_{n}} g_{0}\left(\vec{k}+\vec{q} / 2, i \omega_{n}+i \Omega_{n}\right) g_{0}\left(\vec{k}-\vec{q} / 2, i \Omega_{n}\right) \\
& =\frac{n_{\vec{k}-\vec{q} / 2}-n_{\vec{k}+\vec{q} / 2}}{i \omega_{n}-\vec{k} \cdot \vec{q} / m},
\end{aligned}
$$

and $n_{\vec{k}}=\theta\left(-\xi_{\vec{k}}\right)$ at zero temperature is the free fermion occupation number. $S_{\rho}$ can be expressed in terms of canonical boson fields by introducing

$$
\begin{aligned}
\rho_{\vec{k}}\left(\vec{q}, i \omega_{n}\right)= & \sqrt{\left|\Delta_{\vec{k}}(\vec{q})\right|}\left[\theta\left(\Delta_{\vec{k}}(\vec{q})\right) a_{\vec{k}}^{\dagger}\left(\vec{q}, i \omega_{n}\right)\right. \\
& \left.+\theta\left(-\Delta_{\vec{k}}(\vec{q})\right) a_{\vec{k}}\left(-\vec{q},-i \omega_{n}\right)\right],
\end{aligned}
$$

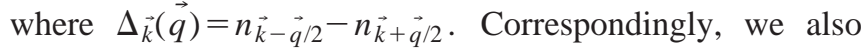
have

$$
\begin{aligned}
\rho_{\vec{k}}\left(-\vec{q},-i \omega_{n}\right)= & \sqrt{\left|\Delta_{\vec{k}}(\vec{q})\right|}\left[\theta\left(\Delta_{\vec{k}}(\vec{q})\right) a_{\vec{k}}\left(\vec{q}, i \omega_{n}\right)\right. \\
& \left.+\theta\left(-\Delta_{\vec{k}}(\vec{q})\right) a_{\vec{k}}^{\dagger}\left(-\vec{q},-i \omega_{n}\right)\right] .
\end{aligned}
$$

Putting Eqs. (9) back into $S_{\rho}$, we obtain after some straightforward manipulations

$$
\begin{aligned}
S_{\rho}= & \frac{1}{2} \sum_{\vec{k}, q, i \omega_{n}}\left(-i \omega_{n}+\frac{|\vec{k} \cdot \vec{q}|}{m}\right) a_{\vec{k}}^{\dagger}\left(\vec{q}, i \omega_{n}\right) a_{\vec{k}}\left(\vec{q}, i \omega_{n}\right) \\
& +\frac{1}{2 L^{d}} \sum_{\vec{k}, \vec{k}^{\prime}, \vec{q}, i \omega_{n}} v(q) \sqrt{\left|\Delta_{\vec{k}}(\vec{q}) \Delta_{\vec{k}^{\prime}}(\vec{q})\right|} \theta\left(\Delta_{\vec{k}}(\vec{q})\right) \\
& \times \theta\left(\Delta_{\overrightarrow{k^{\prime}}}(\vec{q})\right)\left[a_{\vec{k}}^{\dagger}\left(\vec{q}, i \omega_{n}\right) a_{\vec{k}^{\prime}}\left(\vec{q}, i \omega_{n}\right)+a_{\vec{k}}^{\dagger}\left(\vec{q}, i \omega_{n}\right)\right. \\
& \times a_{-\vec{k}^{\prime}}^{+}\left(-\vec{q},-i \omega_{n}\right)+a_{-\vec{k}}\left(-\vec{q},-i \omega_{n}\right) a_{\vec{k}^{\prime}}\left(\vec{q}, i \omega_{n}\right) \\
& \left.+a_{-\vec{k}}\left(-\vec{q},-i \omega_{n}\right) a_{-\vec{k}^{\prime}}^{\dagger}\left(-\vec{q},-i \omega_{n}\right)\right] .
\end{aligned}
$$


Notice that $S_{\rho}$ is an action for interacting bosons described by boson fields $a\left(a^{\dagger}\right) \vec{k}(\vec{q})$ satisfying the usual boson commutation relations $\left[a_{\vec{k}}(\vec{q}), a_{\vec{k}^{\prime}}^{\dagger}\left(\vec{q}^{\prime}\right)\right]=\delta_{\vec{k} \vec{k}^{\prime}} \delta_{q \overrightarrow{q^{\prime}}}$ and $\left[a_{\alpha}, a_{\beta}\right]$ $=\left[a_{\alpha}^{\dagger}, a_{\beta}^{\dagger}\right]=0$. The bosons have kinetic energies $|\vec{k} \cdot \vec{q}| / m$ and interaction terms of the form $\sim v(q)\left(a^{\dagger} a+a^{\dagger} a^{\dagger}+a a\right.$ $\left.+a a^{\dagger}\right)$. In this form the dynamics of the original fermion system is described completely in terms of boson fields (bosonized). Notice that we have so far restricted ourselves to the Gaussian approximation. Higher-order interaction terms between bosons will appear in a cumulant expansion of the $\lambda_{\vec{k}}(\vec{q})$ fields. ${ }^{8}$ The convergence of the cumulant expansion is formally controlled in our model by a small parameter $\epsilon \sim\left(\Lambda / k_{F}\right)^{2(d-1)}$, where a term of order $\left[a^{m} a^{\dagger(n-m)}\right]$ in the cumulant expansion is of order $\epsilon^{n-1}$ because of the appearance of additional integrals of the form

$$
\int d^{d} q v_{e f f}(q, \omega) \times \cdots,
$$

in each increasing order of cumulant expansion, where $v_{\text {eff }}(q, \omega)=v(q) /\left[1-v(q) \chi_{0}(q, \omega)\right]$ is the random-phase approximation (RPA) screened interaction and scales as $\Lambda^{d-2}{ }^{9}$. Notice that the smallness of $\epsilon$ does not guarantee that all calculated physical quantities will converge uniformly in the cumulant expansion. For example, the validity of the Gaussian theory is questionable in the presence of a transverse gauge field. ${ }^{10,11} \mathrm{We}$ shall discuss in more detail the validity of the Gaussian theory in Sec. V. Notice also that, in the $\vec{q} \rightarrow \overrightarrow{0}$ limit, $\Delta_{\vec{k}}(\vec{q}) \rightarrow-\delta\left(\epsilon_{\vec{k}}-\mu\right)(\vec{k} \cdot \vec{q} / m)$ and the usual "tomographic" bosonization procedure based on subdivision of the Fermi surface into disjoint patches at small $\vec{q}$ is recovered. ${ }^{5-7}$ Our bosonization procedure can be viewed as a generalization of the tomographic bosonization method for small wave vector $\vec{q}$ to arbitrary values of $q<\Lambda$.

To understand the nature of the bosonization theory and Gaussian approximation, we first evaluate the free energy associated with $S_{\rho}$. Integrating out the $\rho_{\vec{k}}(\vec{q})$ or $a\left(a^{\dagger}\right) \vec{k}(\vec{q})$ fields in $S_{\rho}$ and using the fact that

$$
\operatorname{Tr}\left[v(q) \hat{\chi}_{0}(\vec{q}, i \omega)\right]^{n}=\left[v(q) \chi_{0}(\vec{q}, i \omega)\right]^{n},
$$

where $\left[\hat{\chi}_{0}(\vec{q}, i \omega)\right]_{\vec{k}, \vec{k}^{\prime}}=\delta_{\vec{k} \vec{k}^{\prime}} \chi_{0 \vec{k}}\left(\vec{q}, i \omega_{n}\right)$ and $\chi_{0}\left(\vec{q}, i \omega_{n}\right)$ is the usual Lindhard function, we obtain

$$
\begin{aligned}
F_{\rho}= & \frac{1}{2 \beta} \sum_{\vec{k}, q, i \omega_{n}} \ln \left[\chi_{0 \vec{k}}\left(\vec{q}, i \omega_{n}\right)\right] \\
& +\frac{1}{2 \beta} \sum_{q, i \omega_{n}} \ln \left(1-v(q) \chi_{0}\left(\vec{q}, i \omega_{n}\right)\right],
\end{aligned}
$$

where the first term in $F_{\rho}$ comes from the kinetic energy of the $a\left(a^{\dagger}\right)$ bosons and the second term is the usual randomphase approximation correction to free energy for interacting fermions. The presence of the RPA term in $F_{\rho}$ suggests that our present Gaussian approximation is essentially the same as RPA theory for interacting fermions, except that the extra "bosonization" assumption in our theory gives rise to the extra kinetic term in $F_{\rho}$. The bosonized action $S_{\rho}$ assumes that the excitations in a fermion system can be fully represented by particle-hole pairs, which are treated as independent bosons in the Gaussian approximation. Note that particle-hole pairs are not all independent in a fermion system because of the Pauli exclusion principle. For example, two particle-hole pairs $f_{\vec{k}}^{\dagger} f_{\vec{p}}$ and $f_{\vec{k}}^{\dagger} f_{\vec{p}^{\prime}}$ are not independent excitations because they both involve creation of fermions in state $\vec{k}$. However, they are treated as independent bosons here as long as $\vec{p} \neq \vec{p}^{\prime}$. Notice, however, that in one dimension the situation becomes different when the fermion spectrum is linearized near the Fermi surface. In this limit the entire particle-hole excitation spectrum can be represented rigorously by bosons ${ }^{5}$ and the Gaussian approximation becomes "exact."

Despite the approximations in the Gaussian theory, the bosonized form of the action has the advantage that within the approximation the full excitation spectrum and the ground and excited state wave functions of the system can be obtained easily. This allows us to study the properties of the system in great detail, as we shall see in the following.

\section{PARTICLE-HOLE EXCITATION SPECTRUM}

The eigenstates and eigenvalue spectrum described by the action $S_{\rho}$ can be obtained by diagonalizing the bosonized action (10) using a generalized Bogoliubov transformation. We introduce for each wave vector $\vec{q}$ the Bogoliubov transformation $^{12}$

$$
\begin{gathered}
a_{\vec{k}}(\vec{q})=\sum_{\vec{k}^{\prime}}\left[\alpha_{\vec{k} \vec{k}^{\prime}} \gamma_{\vec{k}^{\prime}}(\vec{q})+\beta_{\vec{k} \vec{k}^{\prime}} \gamma_{-\vec{k}^{\prime}}^{\dagger}(-\vec{q})\right], \\
a_{-\vec{k}}(-\vec{q})=\sum_{\vec{k}^{\prime}}\left[\alpha_{\vec{k} \vec{k}^{\prime}} \gamma_{-\vec{k}^{\prime}}(-\vec{q})+\beta_{\vec{k} \vec{k}^{\prime}} \gamma_{\vec{k}^{\prime}}^{\dagger}(\vec{q})\right],
\end{gathered}
$$

and correspondingly

$$
\gamma_{\vec{k}}(\vec{q})=\sum_{\vec{k}^{\prime}}\left[\alpha_{\vec{k}^{\prime} \vec{k}}^{*} a_{\vec{k}^{\prime}}(\vec{q})-\beta_{\vec{k}^{\prime} \vec{k}} a_{-\vec{k}^{\prime}}^{\dagger}(-\vec{q})\right],
$$

$$
\gamma_{-\vec{k}}(-\vec{q})=\sum_{\vec{k}^{\prime}}\left[\alpha_{\vec{k}^{\prime} \vec{k}}^{*} a_{-\vec{k}^{\prime}}(-\vec{q})-\beta_{\vec{k}^{\prime} \vec{k}} a_{\vec{k}^{\prime}}^{\dagger}(\vec{q})\right]
$$

where we assume that the $\gamma\left(\gamma^{\dagger}\right) \vec{k}(\vec{q})$ operators diagonize the Hamiltonian, i.e.,

$$
H_{\rho}=\sum_{\vec{k}} E_{\vec{k}}(\vec{q}) \gamma_{\vec{k}}^{\dagger}(\vec{q}) \gamma_{\vec{k}}(\vec{q})+E_{G},
$$

where $E_{\vec{k}}(\vec{q})$ are the eigenenergies and $E_{G}$ is the ground state energy of the system. Notice that a collective mode may appear in the system and is also included in the sum $\Sigma_{\vec{k}}$. The matrix elements $\alpha$ and $\beta$ satisfy the orthonormality condition 


$$
\begin{gathered}
\sum_{\vec{k}^{\prime \prime}}\left[\alpha_{\vec{k} \vec{k}^{\prime \prime}} \alpha_{\vec{k}^{\prime} \vec{k}^{\prime \prime}}^{*}-\beta_{\vec{k} \vec{k}^{\prime \prime}} \beta_{\vec{k}^{\prime} \vec{k}^{\prime \prime}}^{*}\right]=\delta_{\vec{k} \vec{k}^{\prime}}, \\
\sum_{\vec{k}^{\prime \prime}}\left[\alpha_{\vec{k} \vec{k}^{\prime \prime}} \beta_{\vec{k}^{\prime} \vec{k}^{\prime \prime}}-\beta_{\vec{k} \vec{k}^{\prime \prime}} \alpha_{\vec{k}^{\prime} \vec{k}^{\prime \prime}}\right]=0 .
\end{gathered}
$$

Writing down the equations of motion for $a_{\vec{k}}(\vec{q})$ in terms of $\gamma\left(\gamma^{\dagger}\right) \vec{k}(\vec{q}),{ }^{12}$ we obtain the Bogoliubov equations

$$
\begin{aligned}
\left(E_{\overrightarrow{k^{\prime}}}(\vec{q})-\frac{|\vec{k} \cdot \vec{q}|}{m}\right) \alpha_{\vec{k} \vec{k}^{\prime}}= & \frac{v(q)}{L^{d}} \sum_{\vec{k}^{\prime \prime}} \theta\left(\Delta_{\vec{k}}(\vec{q})\right) \theta\left(\Delta_{\overrightarrow{k^{\prime \prime}}}(\vec{q})\right) \\
& \times \sqrt{\left|\Delta_{\vec{k}}(\vec{q}) \Delta_{\vec{k}^{\prime \prime}}(\vec{q})\right|}\left(\alpha_{\vec{k}^{\prime \prime} \vec{k}^{\prime}}+\beta_{\vec{k}^{\prime \prime} \vec{k}^{\prime}}^{*}\right),
\end{aligned}
$$

$$
\begin{aligned}
\left(E_{\overrightarrow{k^{\prime}}}(\vec{q})+\frac{|\vec{k} \cdot \vec{q}|}{m}\right) \beta_{\vec{k} \vec{k}^{\prime}}= & -\frac{v(q)}{L^{d}} \sum_{\vec{k}^{\prime \prime}} \theta\left(\Delta_{\vec{k}}(\vec{q})\right) \theta\left(\Delta_{\vec{k}^{\prime \prime}}(\vec{q})\right) \\
& \times \sqrt{\left|\Delta_{\vec{k}}(\vec{q}) \Delta_{\vec{k}^{\prime \prime}}(\vec{q})\right|}\left(\alpha_{\vec{k}^{\prime \prime} \vec{k}^{\prime}}^{*}+\beta_{\vec{k}^{\prime \prime} \vec{k}^{\prime}}\right) .
\end{aligned}
$$

In general we find that there exist two kinds of solution to these equations: (i) particle-hole continuum, with $E_{\vec{k}}(\vec{q})$ $=|\vec{k} \cdot \vec{q}| / m$, and (ii) collective modes, with energy $E_{0}(\vec{q})$ outside the particle-hole continuum satisfying the RPA eigenvalue equation $1-v(q) \chi_{0}\left(\vec{q}, E_{0}(\vec{q})\right)=0$. Most of the detailed mathematics can be found in Ref. 12. We obtain finally

$$
\begin{aligned}
& \alpha_{\vec{k} \vec{k}^{\prime}}=\delta_{\vec{k} \vec{k}^{\prime}}+P \frac{\theta\left(\Delta_{\vec{k}}(\vec{q})\right) \theta\left(\Delta_{\vec{k}^{\prime}}(\vec{q})\right) \sqrt{\left|\Delta_{\vec{k}}(\vec{q}) \Delta_{\vec{k}^{\prime}}(\vec{q})\right|} v_{e f f}\left(q,\left|\vec{k}^{\prime} \cdot \vec{q}\right| / m\right)}{L^{d}\left(\left|\vec{k}^{\prime} \cdot \vec{q}\right| / m-|\vec{k} \cdot \vec{q}| / m\right)}, \\
& \beta_{\vec{k} \vec{k}^{\prime}}=\frac{-\theta\left(\Delta_{\vec{k}}(\vec{q})\right) \theta\left(\Delta_{\vec{k}^{\prime}}(\vec{q})\right) \sqrt{\left|\Delta_{\vec{k}}(\vec{q}) \Delta_{\vec{k}^{\prime}}(\vec{q})\right|} v_{e f f}\left(q,-\left|\vec{k}^{\prime} \cdot \vec{q}\right| / m\right)}{L^{d}\left(\left|\vec{k}^{\prime} \cdot \vec{q}\right| / m+|\vec{k} \cdot \vec{q}| / m\right)}
\end{aligned}
$$

for the particle-hole continuum spectrum $\vec{k}^{\prime}$, and

$$
\alpha_{\vec{k} 0}=\frac{1}{L^{d / 2}} \frac{\theta\left(\Delta_{\vec{k}}(\vec{q})\right) \sqrt{\left|\Delta_{\vec{k}}(\vec{q})\right|}}{\left[E_{0}(\vec{q})-|\vec{k} \cdot \vec{q}| / m\right]\left[-\partial \chi_{0}(q, \omega) / \partial \omega\right]_{\omega=E_{0}(\vec{q})}^{1 / 2}},
$$

$$
\beta_{\vec{k} 0}=-\frac{1}{L^{d / 2}} \frac{\theta\left(\Delta_{\vec{k}}(\vec{q})\right) \sqrt{\left|\Delta_{\vec{k}}(\vec{q})\right|}}{\left[E_{0}(\vec{q})+|\vec{k} \cdot \vec{q}| / m\right]\left[-\partial \chi_{0}(q, \omega) / \partial \omega\right]_{\omega=E_{0}(\vec{q})}^{1 / 2}},
$$

for the collective mode $E_{0}(\vec{q})$. Notice that in the boson representation the collective mode comes from nonperturbative effects due to interaction and cannot be obtained from analytic continuation of the noninteracting boson modes.

Next we examine the solutions of the bosonized Hamiltonian in the $e^{2} \rightarrow \infty$ limit. First we consider the collective mode. Using the result that $\chi_{0}(\vec{q}, \omega) \rightarrow n_{0} q^{2} / m \omega^{2}$ in the limit $\omega \gg k_{F} q / m$, where $n_{0}$ is the fermion density, ${ }^{13}$ it is easy to see that in the limit $e^{2} \rightarrow \infty$ the collective mode frequencies are given by $E_{0}(q)=\omega_{P}$, where $\omega_{P}=\left(4 \pi n_{0} e^{2} / m\right)^{1 / 2}$ is the plasma frequency. Notice that $\omega_{P} \rightarrow \infty$ as $e^{2} \rightarrow \infty$, indicating that plasma oscillations are outside the physical spectrum in this limit.

Despite the vanishing of collective excitation in the physical spectrum, the particle-hole excitation spectrum with excitation energies $|\vec{k} \cdot \vec{q}| / m$ survives in bosonization theory in the limit $e^{2} \rightarrow \infty$. In this limit $v_{\text {eff }}\left(q,\left|\vec{k}^{\prime} \cdot \vec{q}\right| / m\right)$ $\rightarrow-1 / \chi_{0}\left(q,\left|\vec{k}^{\prime} \cdot \vec{q}\right| / m\right)$ and the coefficients $\alpha_{\vec{k} \vec{k}^{\prime}}$ and $\beta_{\vec{k} \vec{k}^{\prime}}$ remain regular, indicating that the particle-hole excitation spectrum is not qualitatively modified by the effect of confinement. It is instructive to examine the charge fluctuations carried by the particle-hole excitations by examining the commutator $\left[\rho(\vec{q}), \gamma_{\vec{k}}^{\dagger}\left(\vec{q}^{\prime}\right)\right]$. In particular, we expect that the commutator should go to zero in the $e^{2} \rightarrow \infty$ limit, where charge fluctuations are forbidden. Using Eqs. (9), (13), and (16a), and the usual boson commutation rules, it is straightforward to show that

$$
\begin{aligned}
{\left[\rho(\vec{q}), \gamma_{\vec{k}}^{\dagger}\left(-\vec{q}^{\prime}\right)\right]=} & \delta_{\vec{q} \vec{q}^{\prime}} \theta\left(\Delta_{\vec{k}}(\vec{q})\right) \sqrt{\left|\Delta_{\vec{k}}(\vec{q})\right|} \\
& \times \frac{1}{1-v(q) \chi_{0}(\vec{q},|\vec{k} \cdot \vec{q}| / m)},
\end{aligned}
$$

and vanishes in the limit $e^{2} \rightarrow \infty$.

Before ending this section let us examine our results obtained so far from bosonization theory. Within the Gaussian approximation, we obtain a RPA-like excitation spectrum with both collective modes and particle-hole excitations. As the coupling constant $e^{2}$ increases, the energy of the collective mode rises continuously to infinity whereas the particlehole excitation spectrum remains unaltered. The charge fluctuations carried by the particle-hole excitations are projected out gradually as $e^{2}$ increases, resulting in chargeless particle-hole excitations at $q<\Lambda$ in the confinement limit $e^{2} \rightarrow \infty$. Notice that within the Gaussian approximation the confinement state analytically continues to the usual Fermi liquid state and there is no phase transition in between. The theory thus suggests that the confinement state of a gas of fermions is a Fermi liquid state, with, however, chargeless quasiparticles constituting the Fermi liquid. It also suggests that this is a rather unusual Fermi liquid state, since bare 
fermions in the system carry charge $e$, and the quasiparticles must have vanishing overlap with bare fermions if they carry zero charge.

\section{SINGLE-PARTICLE PROPERTIES AND LOW-ENERGY EFFECTIVE LAGRANGIAN}

In the usual bosonization theory for one-dimensional systems, the single-particle properties of the system can be determined once a rigorous representation of the single-particle operator in terms of density operators $\rho_{L}(q)$ and $\rho_{R}(q)$ is obtained. ${ }^{5}$ In higher dimensions, this procedure becomes inadequate for two reasons. (1) The corresponding procedure requires that the bosonized representation of the singleparticle operator $\psi_{b}(\vec{r})$ satisfies the commutation relations

$$
\left[\psi_{b}(\vec{r}), \rho_{\vec{k}}(\vec{q})\right]=e^{-i(\vec{k}+\vec{q} / 2) \cdot \vec{r}} \int d^{d} r^{\prime} e^{i(\vec{k}-\vec{q} / 2) \cdot \vec{r}^{\prime}} \psi_{b}\left(\vec{r}^{\prime}\right)
$$

for all possible momenta $\vec{k}$ and $\vec{q}$. We have not been able to find a representation that satisfies this criterion, ${ }^{6-8}$ and even if we can find such a representation, the theory is still approximate because in dimensions higher than one, the boson representation using Wigner operators is not exact and violates the Pauli exclusion principle, at least in Gaussian approximation. (2) More importantly, unlike in one dimension where the elementary excitations are collective density waves, we have seen in the last section that in dimensions higher than 1 the particle-hole excitation spectrum is Fermiliquid-like, implying that fermionic quasi-particles exist in dimensions higher than one. It is thus important to construct the quasiparticle operators directly in this case.

To construct the quasiparticle operators we first consider the equation of motion of the bare fermion operator $\psi(\vec{r})$ $=\left(1 / L^{d}\right) \sum_{\vec{k}} e^{-i \vec{k} \cdot \vec{r}} f_{\vec{k}}$ at imaginary time,

$$
\begin{aligned}
\frac{\partial \psi(\vec{r})}{\partial \tau}= & \frac{1}{2 m} \nabla^{2} \psi(\vec{r})-\frac{1}{L^{d}} \sum_{\vec{q}} v(q) \rho(\vec{q}) e^{i \vec{q} \cdot \vec{r}} \psi(\vec{r}) \\
= & \frac{1}{2 m} \nabla^{2} \psi(\vec{r})-\frac{1}{L^{d}} \sum_{\vec{q}}\left[v(q) \rho_{p h}(\vec{q})\right. \\
& \left.+v(q) \rho_{c}(\vec{q})\right] e^{i \vec{q} \cdot \vec{r}} \psi(\vec{r}),
\end{aligned}
$$

where

$$
\begin{aligned}
v(q) \rho_{p h}(q)= & \sum_{\vec{k}} \sqrt{\left|\Delta_{\vec{k}}(\vec{q})\right|} \theta\left(\Delta_{\vec{k}}(\vec{q})\right) \\
& \times\left[v_{e f f}\left(q, \frac{-|\vec{k} \cdot \vec{q}|}{m}\right) \gamma_{\vec{k}}^{\dagger}(\vec{q})\right. \\
& \left.+v_{e f f}\left(q, \frac{|\vec{k} \cdot \vec{q}|}{m}\right) \gamma_{-\vec{k}}(-\vec{q})\right], \\
v(q) \rho_{c}(\vec{q})= & L^{d / 2}\left(-\frac{\partial \chi_{0}(q, \omega)}{\partial \omega}\right)_{\omega=E_{0}(\vec{q})}^{-1 / 2} \\
& \times\left[\gamma_{0}^{\dagger}(\vec{q})+\gamma_{0}(-\vec{q})\right],
\end{aligned}
$$

$v(q) \rho_{p h}(\vec{q}) \sim \gamma_{\vec{k}}^{\dagger}(\vec{q})+\gamma_{-\vec{k}}(-\vec{q})$ describes the coupling of the particle-hole excitations to the fermion operator, and $v(q) \rho_{c}(\vec{q}) \sim \gamma_{0}^{\dagger}(\vec{q})+\gamma_{0}(-\vec{q})$ describes the coupling of the collective excitations (plasmons) to the fermion operator. In the $e^{2} \rightarrow \infty$ limit, $\rho_{p h}(q) \sim e^{-2}$ and the term $v(q) \rho_{p h}(q)$ is regular and finite. The strong confinement effect shows up only in the interaction between fermions and collective mode excitations (plasmons). The effect of plasmons on the oneparticle properties can be estimated perturbatively by evaluating the plasmon contribution to the fermion self-energy $\Sigma$ and renormalization factor $z$ to second order using Eqs. (18) and (19). We find that $\Sigma \sim \int_{L^{-1}}^{\Lambda} d^{d} q v(q)$, and $z \sim \partial \Sigma / \partial \omega$ $\sim \Sigma / \omega_{P}$. Notice that both $\Sigma$ and $z$ go to infinity as $e^{2} \rightarrow \infty$. Furthermore, the integrals carry also infrared divergence at dimensions $d \leqslant 2$ for any finite $e^{2}$. The divergence of $z$ at $d=2$ for finite $e^{2}$ has been interpreted as the signature of a marginal Fermi liquid. ${ }^{14}$

The divergence in single-particle self-energy indicates that the bare fermion is not a good starting point for constructing quasiparticle operators. To find a better starting point we first look for a canonical transformation for the single-particle operator that diagonalizes the interaction term between fermions and plasmons. The kinetic energy of fermions and the interaction with particle-hole excitations will be treated afterward. We obtain ${ }^{15}$

$$
\psi_{Q}(\vec{r})=e^{\phi(\vec{r})} \psi(\vec{r}),
$$

where

$$
\phi(\vec{r})=\frac{1}{L^{d / 2}} \sum_{\vec{q}} \frac{e^{i \vec{q}} \cdot \vec{r}}{E_{0}(\vec{q})} \frac{\left[\gamma_{0}^{\dagger}(\vec{q})-\gamma_{0}(-\vec{q})\right]}{\left[-\partial \chi_{0}(q, \omega) / \partial \omega\right]^{1 / 2}},
$$

which represents a fermion operator "dressed" by plasmon modes. It is straightforward to derive the equation of motion of the dressed fermion $\psi_{Q}$,

$$
\begin{aligned}
\frac{\partial \psi_{Q}(\vec{r})}{\partial \tau}= & \frac{1}{2 m}\left(\nabla^{2} \psi_{Q}(\vec{r})-\left\{\nabla^{2} \phi(\vec{r})-:[\nabla \phi(\vec{r})]^{2}:\right] \psi_{Q}(\vec{r})\right. \\
& \left.-2 \nabla \phi(\vec{r}) \cdot \nabla \psi_{Q}(\vec{r})\right) \\
& -\frac{1}{L^{d}} \sum_{\vec{q}} v(q) \rho_{p h}(\vec{q}) e^{i \vec{q} \cdot \vec{r}} \psi_{Q}(\vec{r}),
\end{aligned}
$$

where we have used the results $\left[H, \gamma_{0}(\vec{q})\right]=-E_{0}(\vec{q}) \gamma_{0}(\vec{q})$ and $\left[\gamma_{\vec{k}}(\vec{q}), \gamma_{0}^{\dagger}(\vec{q})\right]=0$, etc., for $\vec{k} \neq 0$ and the usual commutator between bare fermions and density operators to derive the above equation. We have also neglected constant energy terms coming from normal ordering of operators in Eq. (21). It is clear from Eq. (21) that the direct coupling between fermions and plasmons is eliminated in the equation of motion of $\psi_{Q}(\vec{r})$. However, interaction between fermions and particle-hole excitations remains in the equation of motion. Moreover, indirect coupling to the plasmons is also generated from the dressed fermion kinetic energy term as in the similar small polaron problem. ${ }^{15}$ It is easy to see by direct power counting of $e^{2}$ in the $\phi(\vec{r})$ field that the coupling of the dressed fermion to plasmons through the kinetic energy term is much weaker than the original fermion-plasmon cou- 
pling. In particular, we find that the self-energy correction of the dressed fermions from $\phi(\vec{r})$ fields remains finite in the limit $e^{2} \rightarrow \infty$. It is also straightforward to show that the infrared divergence in fermion self-energy in two dimensions is removed for the dressed fermions.

These results suggest that the dressed fermion operators $\psi_{Q}(\vec{r})$ constitute a valid starting point to construct chargeless quasiparticles in the $e^{2} \rightarrow \infty$ limit. The other interaction effects can be treated perturbatively. To show that this is indeed the case we first consider the commutation relation between charge and dressed fermion operators. It is straightforward to show that

$$
\begin{aligned}
{\left[\rho(\vec{q}), \psi_{Q}(\vec{r})\right]=} & \left(\frac{2 \chi_{0}\left(q, E_{0}(\vec{q})\right)}{E_{0}(\vec{q})\left[-\partial \chi_{0}(q, \omega) / \partial \omega\right]_{\omega=E_{0}(\vec{q})}}-1\right) \\
& \times e^{-i \vec{q} \cdot \vec{r}} \psi_{Q}(\vec{r}),
\end{aligned}
$$

which vanishes in the limit $e^{2} \rightarrow \infty$, when $E_{0}(\vec{q}) \rightarrow \omega_{P} \rightarrow \infty$, indicating that the dressed single particle operators $\psi_{Q}(\vec{r})$ are indeed chargeless in the $e^{2} \rightarrow \infty$ limit. To show that $\psi_{Q}(\vec{r})$ and $\psi_{Q}\left(\vec{r}^{\prime}\right)$ define independent quasiparticles when $\vec{r} \neq \vec{r}^{\prime}$ we check the commutation relation between the dressed fermion operators themselves. We obtain

$$
\left[\psi_{Q}(\vec{r}), \psi_{Q}^{\dagger}\left(\vec{r}^{\prime}\right)\right] \sim \frac{1}{n_{0}\left(\pi\left|\vec{r}-\vec{r}^{\prime}\right|\right)^{d-1}} \hat{O}\left(\vec{r}, \vec{r}^{\prime}\right),
$$

in the limits $e^{2} \rightarrow \infty$ and $\left|\vec{r}-\vec{r}^{\prime}\right| \rightarrow \infty$, where

$$
\begin{aligned}
\hat{O}= & \left(\frac{\vec{r}-\vec{r}^{\prime}}{\left|\vec{r}-\vec{r}^{\prime}\right|}\right) \cdot\left\{\psi^{\dagger}\left(\vec{r}^{\prime}\right) e^{\phi(\vec{r})}[\nabla \psi(\vec{r})] e^{\phi^{\dagger}\left(\vec{r}^{\prime}\right)}\right. \\
& \left.-\left[\nabla \psi^{\dagger}\left(\vec{r}^{\prime}\right)\right] e^{\phi(\vec{r})} \psi(\vec{r}) e^{\phi^{\dagger}\left(\vec{r}^{\prime}\right)}\right\} .
\end{aligned}
$$

The vanishing of the commutator between different $\psi_{Q}$ operators separated by large distances at dimensions larger than 1 indicates that they can be used to construct independent quasiparticle operators when describing the dynamics of these systems at long distance. Notice that in one dimension such a construction is not possible because of the long rangeness of the commutation relation. In fact, the only fermionic operators that commute with density operators $\rho(\vec{q})$ are the ladder operators ${ }^{5}$ which raise or lower the number of particles in the system by 1 . There are only two independent ladder operators in the system; they change the number of left-going and right-going fermions and cannot be used to construct local quasiparticle excitations.

We shall now proceed to write down an effective action for our quasiparticle operator $\psi_{Q}(\vec{r})$ in the projected Hilbert space $\rho(q)|\psi\rangle=0$, assuming that they can be treated as canonical fermions. The effective action is constructed with the requirement that it reproduces the equation of motion (21) for the quasiparticle operator $\psi_{Q}$. It is straightforward to verify that the correct action is

$$
\begin{aligned}
S_{e f f}\left(\psi_{Q}, \psi_{Q}^{\dagger}\right)= & \int_{0}^{\beta} d \tau d^{d} x \psi_{Q}^{\dagger}(\vec{x}, t) \\
& \times\left(\frac{\partial}{\partial \tau}-\frac{\nabla^{2}}{2 m}-\mu+\phi_{e f f}(\vec{x}, t)\right) \psi_{Q}(\vec{x}, t),
\end{aligned}
$$

where $\phi_{e f f}(\vec{x}, t) \sim v(q) \rho_{p h}(q)$, and with dynamics given by

$$
S_{e f f}(\phi)=-\frac{1}{2} \sum_{q<\Lambda, \omega} \chi_{0}(q, \omega)\left|\phi_{e f f}(q, \omega)\right|^{2} .
$$

We have neglected the plasmon field contributions to the dynamics of $\psi_{Q}$ in $S_{e f f}\left(\psi_{Q}, \psi_{Q}^{\dagger}\right)$. Notice that the contructed effective action is valid in both two and three dimensions where quasiparticle operators can be constructed. In particular, because of the regularity of the interaction between fermions in $S_{e f f}$, it is expected that a Fermi liquid state of chargeless fermions $\psi_{Q}$ will be formed in both dimensions. The residual plasmon interaction induced a weak shortranged effective attractive interaction between $\psi_{Q}$ 's and may lead to superconductivity. We shall not discuss this possibility in this paper.

Finally, assuming that the plasmons can be treated as independent excitations, we obtain to leading order in the projected Hilbert space,

$$
\begin{aligned}
\left\langle\psi^{\dagger}(\vec{r}) \psi\left(\vec{r}^{\prime}\right)\right\rangle \sim & \exp \left[-2\left(\pi m e^{2} / n_{0}\right)^{1 / 2} \int_{L^{-1}}^{\Lambda} d^{d} q \frac{1}{q^{2}}\right. \\
& \left.\times\left\{1-\cos \left[\vec{q} \cdot\left(\vec{r}-\vec{r}^{\prime}\right)\right]\right\}\right]\left\langle\psi_{Q}^{\dagger}(\vec{r}) \psi_{Q}\left(\vec{r}^{\prime}\right)\right\rangle .
\end{aligned}
$$

In particular, the average density of bare fermions and dressed fermions is the same and the Fermi surface volume of the dressed fermions is exactly the same as that of the bare fermions. It is also easy to show that

$$
\begin{aligned}
\left\langle\psi_{Q}^{\dagger}(\vec{r}) \psi(\vec{r})\right\rangle \sim & \exp -\left[\left(\frac{\pi m e^{2}}{n_{0}}\right)^{1 / 2}\left(\int_{L^{-1}}^{\Lambda} d^{d} q \frac{1}{q^{2}}\right)\right] \\
& \times\left\langle\psi^{\dagger}(\vec{r}) \psi(\vec{r})\right\rangle,
\end{aligned}
$$

which vanishes in the $e \rightarrow \infty$ limit, indicating that the bare fermions and dressed fermions have zero wave function overlap, as is expected on physical grounds. Using Eq. (24a), it is straightforward to show that the bare fermion occupation number $n(\vec{k})$ has no discontinuity across the Fermi surface in the $e^{2} \rightarrow \infty$ limit (3D), in agreement with the marginal Fermi liquid picture.

\section{SUMMARY}

Using a bosonization approximation we studied in this paper a gas of fermions interacting via a scalar potential $v(q)=4 \pi e^{2} / q^{2}$ for $q<\Lambda \ll k_{F}$. In particular, we considered the $e^{2} \rightarrow \infty$ limit where the potential becomes confining. We note that, because of the low-momentum cutoff $\Lambda \ll k_{F}$ in our model, formation of a Wigner crystal state is not warranted 
and the system may remain in a liquid state. In this case a different treatment of the problem is necessary. Within a Gaussian approximation we obtain a liquid state solution where the particle-hole excitation spectrum of the system is always Fermi-liquid-like, with the charge carried by the particle-hole excitation vanishing continuously in the $e^{2}$ $\rightarrow \infty$ limit. Moreover, chargeless fermionic operators are constructed that can be used as the starting point for constructing quasiparticles in the system. The solution we obtained describes a marginal Fermi liquid state where the chargeless quasiparticles have vanishing wave function overlap with bare fermions in the system.

An assumption we have made in our analysis is that the Gaussian approximation for the Wigner bosons describes at least qualitatively correctly the particle-hole and collective excitation spectra of the system. The validity of this assumption has been questioned in the case of marginal fermi liquids where the Fermi surface discontinuity $z$ approaches zero. ${ }^{11}$ It was pointed out that the vanishing of $z$ may invalidate the (Gaussian) bosonization approximation, which assumes a finite Fermi surface discontinuity to start with. ${ }^{11}$ In three dimensions, $z$ is finite as long as $e^{2}$ is finite, and vanishes only in the limit $e^{2} \rightarrow \infty$. Thus, our bosonization approximation should be qualitatively valid at any finite $e^{2}$, and can be continued to the $e^{2} \rightarrow \infty$ limit as long as the system evolves adiabatically to this limit.

The situation is more complicated in two dimensions. It was pointed out by Bares and $\mathrm{Wen}^{14}$ that fermion systems interacting with an $e^{2} / q^{2}$ potential (with finite $e^{2}$ ) are marginal Fermi liquids because of the orthogonality catastrophe effect associated with adding particles (charges) to the system. However, the spectrum of particle-hole excitations is not affected by the orthogonality catastrophe, and the collective excitation spectrum of the system can be described by Gaussian theory with nonsingular higher-order corrections, although the underlying quasiparticles that constitute the particle-hole excitation spectrum are orthogonal to the noninteracting fermions. We believe that the same conclusion can be reached in our system as long as the marginal Fermi liquid picture of Bares and Wen evolves adiabatically to the $e^{2} \rightarrow \infty$ limit. The marginal fermi liquid picture in two dimensions can be justified by a renormalization group approach in momentum space, ${ }^{14,18}$ where we reduce the cutoff $\Lambda$ in the cumulant expansion progressively. ${ }^{18}$ To one-loop order, we find that additional short-ranged interactions between fermions are generated in the process of renormalization, with the interaction remaining finite in the limit $e^{2}$ $\rightarrow \infty$ because of screening. ${ }^{16}$ However, the long-ranged Coulomb interaction remains unrenormalized. The unrenormalized Coulomb interaction leads to marginal Fermi liquid behavior which is reflected in the vanishing of the renormalization factor $z .{ }^{14}$ However, the Landau Fermi liq- uid parameters are finite, indicating that the system is a stable (marginal) Fermi liquid.

Notice that although the eigen-particle-hole excitations in our theory [Eq. (16a)] are derived in Gaussian theory and are approximate, the plasmon excitations described by Eq. (16b) are exact in the $q \rightarrow 0$ or $e^{2} \rightarrow \infty$ limits. In particular, our canonical transformation (20) for the dressed fermions $\psi_{Q}$ involves only the plasmon operator and is independent of the Gaussian approximation. The effective action $S_{\text {eff }}\left(\psi_{Q}, \psi_{Q}^{\dagger}\right)$ that we obtained for the dressed fermions describes scattering of dressed fermions with particle-hole excitations via a non-singular short-range potential, which suggests strongly that the dressed fermions form a Fermi liquid state, in agreement with the marginal Fermi liquid picture obtained from (Gaussian) bosonization theory.

It is interesting to make comparison between our approach and the usual treatment of the longitudinal $U(1)$ gauge field in the $t$ - $J$ model where the gauge field imposes the constraint $\rho_{s}(q)+\rho_{h}(q)=0,{ }^{17}$ where $\rho_{s}$ and $\rho_{h}$ are the spin and hole densities, respectively. In the usual treatment, ${ }^{17}$ the spins and holes are integrated out at the Gaussian level, resulting in an effective action for the longitudinal gauge field $\phi_{\text {eff }}$

$$
S_{e f f} \sim-\frac{1}{2}\left[\chi_{0 s}(q, \omega)+\chi_{0 h}(q, \omega)\right]\left|\phi_{e f f}(q, \omega)\right|^{2},
$$

where $\chi_{0 s}(q, \omega)$ and $\chi_{0 h}(q, \omega)$ are the free spin and hole density-density response functions, respectively. It is then assumed that the spinons and holons in the projected Hilbert space can be treated as free particles interacting with the gauge fields with dynamics given by $S_{\text {eff }}{ }^{17}$ Although our analysis here includes only fermions and cannot be directly applied to the $t-J$ model, the form of our low-energy effective Lagrangian is in agreement with this assumption. We shall address this problem more carefully in a future paper. Notice also that we have considered here only the effect of longitudinal gauge fields in a gas of fermions. Similar physics is expected to be found in the presence of a transverse gauge field. In the $e^{2} \rightarrow \infty$ limit, we expect that the chargeless quasiparticles we construct will decouple from the gauge fields and form a (marginal) Fermi liquid. However, it is easy to show that the $e^{i \phi(\vec{r})}$ operator we have constructed is not able to "screen out" the transverse gauge field because it is constructed from density fluctuations, which couple to the longitudinal gauge field only. The transverse gauge field needs a separate treatment and we shall discuss it in a future paper.

\section{ACKNOWLEDGMENTS}

The author thanks Z.-B. Su and N. Nagaosa for helpful questions and comments. This work is supported by HKUGC through RGC Grant No. HKUST6124/98P.

\footnotetext{
${ }^{1}$ N. Nagaosa and P.A. Lee, Phys. Rev. Lett. 60, 2450 (1990); X.G. Wen and P.A. Lee, ibid. 76, 503 (1996).

${ }^{2}$ B.I. Halperin, P.A. Lee, and N. Read, Phys. Rev. B 47, 7312 (1993); Y.B. Kim, P.A. Lee, and X.G. Wen, ibid. 52, 17275 (1995).
}

\footnotetext{
${ }^{3}$ See A. Stern, B.I. Halperin, F. von Oppen, and S.H. Simon, cond-mat/9812135 (unpublished) for a review.

${ }^{4}$ S. Tomonaga, Prog. Theor. Phys. 5, 544 (1950); D.C. Mattis and E.H. Lieb, J. Math. Phys. 6, 304 (1965).

${ }^{5}$ See, for example, F.D.M. Haldane, J. Phys. C 14, 2585 (1981).
} 
${ }^{6}$ F.D.M. Haldane, Helv. Phys. Acta 65, 152 (1992).

${ }^{7}$ A. Houghton and J.B. Marston, Phys. Rev. B 48, 7790 (1993); A.H. Castro Neto and E. Fradkin, Phys. Rev. Lett. 72, 1393 (1994)

${ }^{8}$ P. Kopietz, J. Hermisson, and K. Schönhammer, Phys. Rev. B 52, 10877 (1995).

${ }^{9}$ C. Castellani and C. Di Castro, Physica C 235-240, 99 (1994); C. Castellani, C. Di Castro, and A. Maccarone, Phys. Rev. B 55, 2676 (1997).

${ }^{10}$ D.V. Khveshchenko and P.C.E. Stamp, Phys. Rev. Lett. 71, 2118 (1993); see also B.L. Altshuler, L.B. Ioffe, and A.J. Millis, Phys. Rev. B 50, 14048 (1994).
${ }^{11}$ H.J. Kwon, A. Houghton, and J.B. Marston, Phys. Rev. B 52, 8002 (1995).

${ }^{12}$ A.H. Castro Neto and E. Fradkin, Phys. Rev. B 49, 10877 (1994).

${ }^{13}$ See, for example, G.D. Mahan, Many-Particle Physics (Plenum Press, New York, London (1990).

${ }^{14}$ P.A. Bares and X.G. Wen, Phys. Rev. B 48, 8636 (1993).

${ }^{15}$ Notice that a similar result is also obtained in the case of the small polaron problem; see Ref. 8 for example.

${ }^{16}$ T.K. Ng (unpublished).

${ }^{17}$ P.A. Lee and N. Nagaosa, Phys. Rev. B 46, 5621 (1992).

${ }^{18}$ R. Shankar, Rev. Mod. Phys. 66, 129 (1994). 